\title{
Colon Capsule Endoscopy: An Alternative for Conventional Colonoscopy?
}

\author{
Britt B.S.L. Houwen and Evelien Dekker \\ Department of Gastroenterology and Hepatology, Amsterdam University Medical Centers, Location Academic Medical Center, \\ University of Amsterdam, Amsterdam, The Netherlands
}

See "Colon Capsule Endoscopy: Indications, Findings, and Complications - Data from a Prospective German Colon Capsule Registry Trial (DEKOR)” by Johannes Hausmann, Andrea Tal, Artur Gomer, et al., on page 92-99.

To increase the screening numbers among patients unable or reluctant to undergo conventional colonoscopy alternative screening modalities might be offered. Colon capsule endoscopy (CCE) is a widely used non-invasive imaging, radiation-free method for directly visualizing colon and rectum. ${ }^{1}$ CCE was initially met with skepticism, because of the need for extensive bowel cleansing protocols, long evaluation time, impossibility to take biopsies and remove polyps and high procedural costs. Concerning polyp and cancer detection, several prospective studies have compared CCE with conventional colonoscopy. In these studies, sensitivity and specificity for identifying colonic lesions with CCE was relatively low in comparison with colonoscopy. ${ }^{2-4}$

In this issue of Clinical Endoscopy, Hausmann et al. attempted to provide real-world data on CCE in a large prospective German cohort. ${ }^{5}$ In this registry trial, CCE (CCE-2) was performed in patients unable or unwilling to undergo colonoscopy that was indicated for various reasons. A total of 161 patients underwent CCE at six German centers between 2010

\footnotetext{
Received: January 11, 2021 Revised: January 16, 2021

Accepted: January 16, 2021

Correspondence: Evelien Dekker

Department of Gastroenterology and Hepatology, Amsterdam University Medical Centers, Location Academic Medical Center, University of Amsterdam, Meibergdreef 9, 1105 AZ Amsterdam, The Netherlands

Tel: +31-20-566-4702, Fax: +31-20-691-7033, E-mail: e.dekker@amsterdamumc. $\mathrm{nl}$

ORCID: https://orcid.org/0000-0002-4363-0745
}

(c) This is an Open Access article distributed under the terms of the Creative Commons Attribution Non-Commercial License (http://creativecommons.org/ licenses/by-nc/3.0) which permits unrestricted non-commercial use, distribution, and reproduction in any medium, provided the original work is properly cited. and 2015 and their data were analyzed. The most frequent clinical indications for CCE among 153 analyzed patients were clinical symptoms $(n=72,47 \%)$ and colorectal cancer (CRC) screening $(n=55,36 \%)$. For those patients, the most frequent reasons for undergoing CCE were the patients' specific request to undergo CCE instead of colonoscopy $(n=85,56 \%)$, and having undergone an incomplete colonoscopy $(n=51,32 \%)$. Visualization of the entire colon was obtained by CCE in $69 \%$ $(n=111)$ of patients. In 52 of 161 CCE investigations (32\%), at least one polyp was detected; 24 (15\%) had a non-diminutive polyp ( $>6 \mathrm{~mm}$ ). In two patients $(1.2 \%)$ the suspicion of an adenocarcinoma was raised, which was histopathologically confirmed after surgery. In one patient, known with inflammatory bowel disease, the capsule was stuck in a benign stenosis of the small intestine and had to be removed surgically.

This study provides us with some insights in the use of CCE in daily practice. We would like to discuss several aspects of CCE. First of all, the success rate of the procedure (i.e., entire visualization of the colon), which still is relatively low at $69 \%$. The reasons for unsuccessful CCE were incomplete small bowel and/or colon passage $(n=33)$, inadequate bowel cleansing $(n=7)$ and technical malfunction $(n=2)$. In those patients having undergone a previous incomplete colonoscopy, it could be interesting to know if the colonic segments that were not reached during the previous colonoscopy were now visualized with CCE, resulting in a full colon examination when adding up the two. If so, CCE might indeed be a valuable option after incomplete colonoscopy, but if this is not the case, computed tomographic colonography (CTC) seems a better alternative. The European Society of Gastrointestinal Endoscopy (ESGE)/ 
European Society of Gastrointestinal and Abdominal Radiology (ESGAR) guideline recommends to consider CTC or CCE to explore proximal colonic segments in the setting of incomplete colonoscopy for non-neoplastic colonic conditions or anatomical reasons. ${ }^{1}$ However, in case of pain or spasms as the main reason for incomplete colonoscopy, repeat colonoscopy with more sedation or CTC is advised.

In this study, although patient age and indications varied widely, polyp and cancer detection rates might seem rather low. In only $33 \%$ of all CCE procedures one or more polyps or cancer were detected. However, as patients were not all subjected to a colonoscopy as well, the diagnostic accuracy of CCE in detecting polyps and cancer could not be assessed. However, comparing the diagnostic accuracy of CCE and CTC with the gold standard colonoscopy is definitely of interest. When reviewing studies published in literature, CCE seems to have a comparable accuracy for polyp detection as CTC. ${ }^{6,7}$ A recent prospective, randomized study showed a higher detection rate for CCE (polyps $\geq 6 \mathrm{~mm}, 32 \%$; and polyps $\geq 10 \mathrm{~mm}, 14 \%$ ) compared to CTC (polyps $\geq 6 \mathrm{~mm}, 9 \%$; and polyps $\geq 10 \mathrm{~mm}, 6 \%)^{6.7}$ Sensitivity of CCE for polyps $\geq 6$ $\mathrm{mm}$ and polyps $\geq 10 \mathrm{~mm}$ ( $84 \%$ and $84 \%$ ) was higher than to that of CTC ( $32 \%$ and $53 \%$, respectively). However, specificity for polyps $\geq 6 \mathrm{~mm}$ was higher for CTC versus CCE ( $99 \%$ vs. $93 \%$, respectively) and comparable for polyps $\geq 10 \mathrm{~mm}$ (99\% vs. $97 \%$, respectively).

One of the disadvantages of CCE, its time-consuming and tedious reading effort, might limit its widespread use. However, reading times may be significantly reduced by recent advancements in machine learning and algorithms and more data on its efficacy are eagerly awaited. ${ }^{8}$ On the other hand, the procedural costs for CCE as a solely diagnostic test are still considerable.

Regarding patient burden, although CCE is non-invasive there is a need of high quality bowel preparation to enable good visualization of polyps, especially if flat or serrated. This bowel preparation exceeds the regimens for normal colonoscopy and CTC and includes a 1- or 2-day bowel cleansing protocol with polyethylene glycol often combined with a booster preparation like sodium phosphate or ascorbic acid, and no solid food intake 1 or 2 days before CCE.

In this study, one third of the enrolled patients ( $n=55,36 \%)$ chose CCE as primary CRC screening method in a country where colonoscopy is the primary screening method. As this was not a randomized study and we are neither informed on how often the option of a screening CCE was offered, we do not know the preference and participation rate of CCE as a primary screening method for CRC in Germany. In Europe however, the European Union recommends fecal immunochemical test (FIT) as the preferred method for popula- tion-based screening programs, followed by colonoscopy in FIT-positives. ${ }^{9}$ Such FIT-based programs have high participation rates, and a positive predictive value of approximately $40 \%$ which makes it not so attractive to follow a positive FIT by another non-invasive test where no therapy nor pathology-based diagnosis is possible. ${ }^{10}$ Therefore, CCE might only be a sensible option for those FIT-positives that are unwilling to undergo colonoscopy.

In other countries or regions, depending on many factors like availability of endoscopy services and finances, the preferred screening methods might be different, and primary screening by colonoscopy may be recommended. In these areas, participation-rates could possibly be increased by offering CCE as an alternative screening method. However, data of prospective studies on aspects like participation rates, accuracy, patient burden, logistics and cost-effectivity are needed first.

\section{Conflicts of Interest}

Evelien Dekker received equipment on loan from Olympus and Fujifilm, Evelien Dekker received a research grant from FujiFilm, a consulting fee for medical advice from Tillots, Olympus, Fujifilm, GI Supply and CPP-FAP and a speakers' fee from Olympus, Roche and GI Supply. Evelien Dekker has been an Editorial Board member of Clinical Endoscopy; however, she was not involved in the peer reviewer selection, evaluation, or decision process of this article. The other author has no potential conflicts of interest.

Funding

None.

Author Contributions

Writing-original draft: Britt B.S.L. Houwen

Writing-review\&editing: BBSLH, Evelien Dekker

ORCID

Britt B.S.L. Houwen:

https://orcid.org/0000-0003-1920-8947

Evelien Dekker: https://orcid.org/0000-0002-4363-0745

\section{REFERENCES}

1. Spada C, Hassan C, Bellini D, et al. Imaging alternatives to colonoscopy: CT colonography and colon capsule. European Society of Gastrointestinal Endoscopy (ESGE) and European Society of Gastrointestinal and Abdominal Radiology (ESGAR) guideline - update 2020. Endoscopy 2020;52:1127-1141.

2. Pecere S, Senore C, Hassan C, et al. Accuracy of colon capsule endoscopy for advanced neoplasia. Gastrointest Endosc 2020;91:406-414.e1.

3. Rex DK, Adler SN, Aisenberg J, et al. Accuracy of capsule colonoscopy in detecting colorectal polyps in a screening population. Gastroenterology 2015;148:948-957.e2.

4. Van Gossum A, Munoz-Navas M, Fernandez-Urien I, et al. Capsule endoscopy versus colonoscopy for the detection of polyps and cancer. $\mathrm{N}$ Engl J Med 2009;361:264-270.

5. Hausmann J, Tal A, Gomer A, et al. Colon capsule endoscopy: indications, findings, and complications - data from a prospective German 
colon capsule registry trial (DEKOR). Clin Endosc 2021;54:92-99.

6. Cash BD, Fleisher MR, Fern S, et al. A multicenter, prospective, randomized study comparing the diagnostic yield of colon capsule endoscopy versus computed tomographic colonography in a screening population. Results of the TOPAZ study. Gastrointest Endosc 2019;89(6 Suppl):AB87-AB88.

7. Spada C, Hassan C, Barbaro B, et al. Colon capsule versus CT colonography in patients with incomplete colonoscopy: a prospective, comparative trial. Gut 2015;64:272-281.

8. Yang YJ. The future of capsule endoscopy: the role of artificial intelli- gence and other technical advancements. Clin Endosc 2020;53:387-394.

9. Săftoiu A, Hassan C, Areia M, et al. Role of gastrointestinal endoscopy in the screening of digestive tract cancers in Europe: European Society of Gastrointestinal Endoscopy (ESGE) position statement. Endoscopy 2020;52:293-304

10. Wieten E, de Klerk CM, van der Steen A, et al. Equivalent accuracy of 2 quantitative fecal immunochemical tests in detecting advanced neoplasia in an organized colorectal cancer screening program. Gastroenterology 2018;155:1392-1399.e5. 